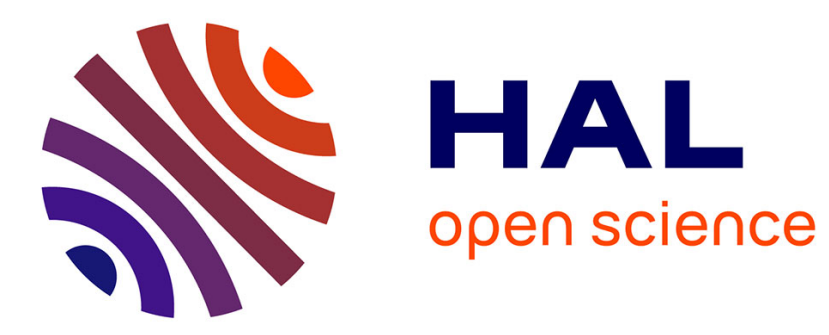

\title{
Optical characterization of ultra-short Bragg grating on lithium niobate ridge waveguide
}

Clément Guyot, Gwenn Ulliac, Jean Dahdah, Wentao Qiu, Maria-Pilar

Bernal, Fadi Issam Baida, Nadège Courjal

\section{To cite this version:}

Clément Guyot, Gwenn Ulliac, Jean Dahdah, Wentao Qiu, Maria-Pilar Bernal, et al.. Optical characterization of ultra-short Bragg grating on lithium niobate ridge waveguide. Optics Letters, 2014, 39, pp.371 - 374. 10.1364/OL.39.000371 . hal-00942573

\section{HAL Id: hal-00942573 \\ https://hal.science/hal-00942573}

Submitted on 6 Feb 2014

HAL is a multi-disciplinary open access archive for the deposit and dissemination of scientific research documents, whether they are published or not. The documents may come from teaching and research institutions in France or abroad, or from public or private research centers.
L'archive ouverte pluridisciplinaire HAL, est destinée au dépôt et à la diffusion de documents scientifiques de niveau recherche, publiés ou non, émanant des établissements d'enseignement et de recherche français ou étrangers, des laboratoires publics ou privés. 


\title{
Optical characterization of ultra-short Bragg grating on lithium niobate ridge waveguide
}

\author{
Clément Guyot ${ }^{1}$, Gwenn Ulliac ${ }^{1}$, Jean Dahdah ${ }^{2}$, Wentao Qiu ${ }^{1}$, Maria-Pilar Bernal ${ }^{1}$, Fadi Baida ${ }^{1}$, and \\ Nadège Courjal ${ }^{1, *}$ \\ ${ }^{1}$ FEMTO-ST Institute UMR6174, University of Franche-Comté, Besançon, France \\ ${ }^{2}$ Kapteos, 23 av. du Lac Leman, Le Bourget du Lac, France \\ *Corresponding author: nadege.courjal@femto-st.fr
}

\begin{abstract}
In this letter, we report a technique to etch giant aspect ratio nanostructures in lithium niobate. A eight micrometer long Bragg grating on a Ti: $\mathrm{LiNbO}_{3}$ ridge waveguide was fabricated by combining optical grade dicing and Focused Ion Beam (FIB) milling. The reflectivity was evaluated using an optical coherence tomography (OCT) system: it is measured to be $53 \%$ for the TM wave and $47 \%$ for the TE wave. We study by 2D-FDTD the modeled behaviour of the electromagnetic field when an angle exists between two consecutive sidewalls of the grating, in order to understand the difference between ideal Bragg grating and experimental samples. These simulations allow us to optimize the parameters in order to increase the reflection of the grating up to $80 \%$.

OCIS Codes: (230.1480) Bragg reflector, (050.5298) Photonic crystals, (130.3730) Lithium niobate.
\end{abstract}

The development of nonlinear or electro-optical microresonators represents a very stimulating challenge to achieve advanced functionalities in compact optical processing devices. Lithium niobate appears to be an excellent candidate for such realizations, due to its well known electro-optic, pyro-electric and nonlinear properties. Microring resonators have been reported recently in sub-micrometric $\mathrm{LiNbO}_{3}$ thin films with high index contrasts fabricated by ion slicing technologies [1]. An alternative approach consists in using high reflectance Bragg gratings as building blocks for Fabry-Perot cavities. This approach allows cavities with lengths as small as $N / 2$, and has motivated numerous studies for the last decades. The quality factor of the Fabry-Perot cavity is strongly related to the Bragg mirrors reflectivity. Several techniques already exist to induce periodic perturbation of the effective refractive index, like photorefraction, protonic exchange, laser femtosecond writing or deposition of surface grating [2-6]. A reflectivity as high as $95 \%$ has been reported, but the grating was written along millimeter distances of waveguide to compensate the small index contrast [2].

Interaction lengths should however be drastically reduced in order to meet the specifications of compact photonic circuits. In our previous work, we showed a 288 $\mu \mathrm{m}$ long Bragg grating with $50 \%$ of reflection. The grating was entirely etched by FIB milling on a standard lithium niobate waveguide [7]. By increasing the index contrast variation, Zhou et al. have reported a $175 \mu \mathrm{m}$ long Bragg grating with a reflectivity of $50 \%$ [8]. The grating was made by photorefraction in ion implanted lithium niobate crystals. To increase the index contrast, a process combining Focused Ion Beam (FIB) milling with wet etching allowed to etch below the waveguide and thus create a thin membrane [8]. In this letter, we propose to go one step further toward extremely short Bragg gratings with an experimental reflectivity of $53 \%$ on a ridge waveguide which was optimized to reduce optical propagation losses. The reflectivity of the grating should be increased up to $80 \%$ by properly adjusting its period. Additionally, we show how an optical coherence tomography system can advantageously be exploited to fully characterize waveguides and reflectors.

Our purpose is to reach a high reflectivity with an ultrasmall pattern (see Fig. 1). The Bragg gratings are made of periodic deep grooves etched in ridge waveguides. In order to get a maximum reflectivity of $99.7 \%$ at $1550 \mathrm{~nm}, 5$ grooves are etched with a period of $1690 \mathrm{~nm}$ and a markto-space ratio of $31.25 \%\left(528 \mathrm{~nm}\right.$ of $\mathrm{LiNbO}_{3}$ and $1162 \mathrm{~nm}$ of air). These parameters are calculated with a transfermatrix method.

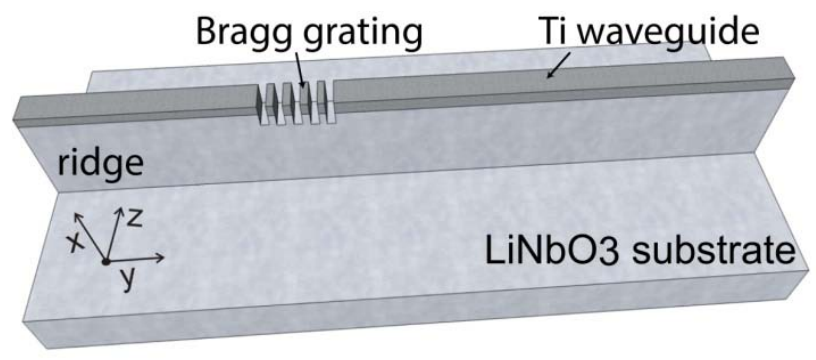

Fig. 1. Scheme of a ridge with a Bragg grating.

The first step consists in fabricating a planar waveguide on X-cut wafer by titanium-indiffusion, that insures the propagation of both TE ( $\mathrm{H}$ field on X-axis) and TM polarized guided modes, compared to proton exchanged (PE) waveguides for which guided modes are more vertically confined but forbid the propagation of one of the polarizations [9]. In order to confine the modes laterally, a ridge structure is realized in a second step by optical grade dicing with a circular precision saw (DISCO DAD 
3350), as shown in Fig. 2. The prepared ridge is $5.6 \mu \mathrm{m}$ wide at its top and $48 \mu \mathrm{m}$ deep. This method described in [10] allows in a simple way to simultaneously cut and polish the lateral sides of the ridge. This confinement is fundamental to enhance electro-optical and non-linear effects [11]. Cutting parameters led to smooth ridges as can be judged by the propagation losses that were estimated to be as low as $1.05 \pm 0.05 \mathrm{~dB} / \mathrm{cm}$ for TE wave and $3.2 \pm 0.1 \mathrm{~dB} / \mathrm{cm}$ for TM wave. The optical losses were estimated with the method described in [12]. Those low propagation losses are necessary to warrant resonances with high quality factors. a.

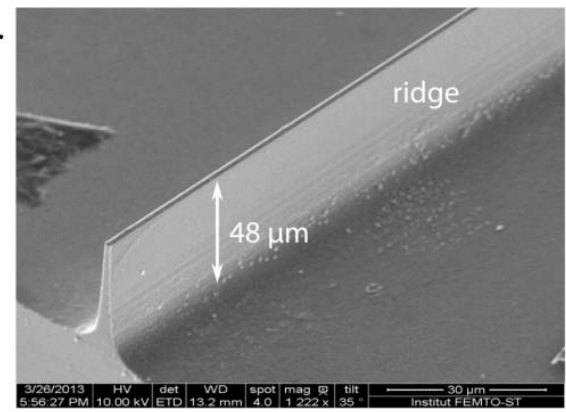

b.

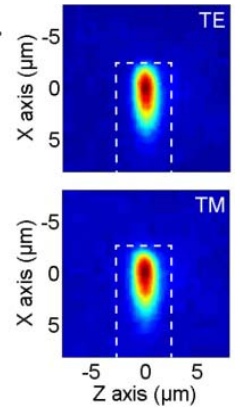

Fig. 2. a. SEM image of a ridge cut by optical grade dicing. b. Visualization of TE and TM modes at $1.55 \mu \mathrm{m}$ with IR camera. The dashed lines represent the limit between the ridge and the air.

Finally, a Bragg grating with 5 air grooves is etched by focused ion beam (FIB) milling on the top of the ridge. This fabrication process offers the ability to etch indifferently from the topside or from the lateral side of the ridge. However, etching from the topside of the ridge ineluctably leads to conical-shaped patterns in the depth of the ridge (Fig. 3.a): the sidewall angle, which corresponds to the angle between two consecutives layers of lithium niobate, is no better than $8^{\circ}$ in that case, which is due to matter redeposition during the etching process [13]. This conical shape induces a major unwanted effect: transmitted and reflected light through the grating sinks into the substrate after a few periods only [14]. As an example, Fig. 3.b shows the modeled distribution calculated by $2 \mathrm{D}$-FDTD of the $\mathrm{E}_{\mathrm{x}}$ field propagating in a two-period Bragg grating etched from the top of lithium niobate waveguide. a.

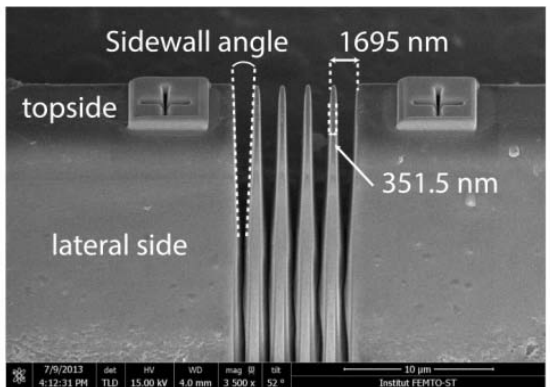

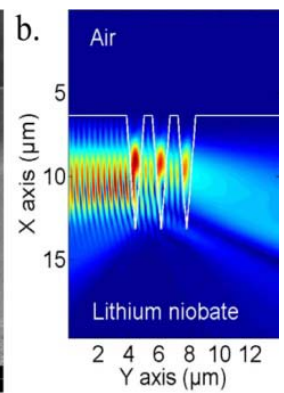

Fig. 3. a. SEM tilted view of a Bragg grating etched by FIB milling from top of a ridge. $b$. Modeled behavior of $\mathrm{E}_{\mathrm{x}}$ field through the grating. The simulation area represents a cross section of the ridge along the direction of propagation.
Otherwise, if the pattern is milled from the lateral side of the ridge, as it is shown in Fig. 4, the aspect ratio is just limited by the length of the lateral trenches: in Fig. 4, the grating shows a depth of $5 \mu \mathrm{m}$, but this depth can be easily increased by etching longer trenches along the sidewall. Now the angle between two consecutive walls is reduced from $8^{\circ}$ to $3.8^{\circ}$. Indeed the FIB beam can go through the entire width of the ridge, which limits the matter redeposition during the etching. Consequently, this technological process gives the opportunity to fabricate gratings with quasi-parallel trenches (sidewall angles along the width of the ridge $<4^{\circ}$ ) together with giant aspect ratios. Besides, the trenches are completely vertical along the depth. This Bragg grating was etched in less than two hours with a probe current of $230 \mathrm{pA}$, a writefield of $50 \mu \mathrm{m}$, a step size of $10 \mathrm{~nm}$, and a dwell-time of $0.1 \mathrm{~ms}$.

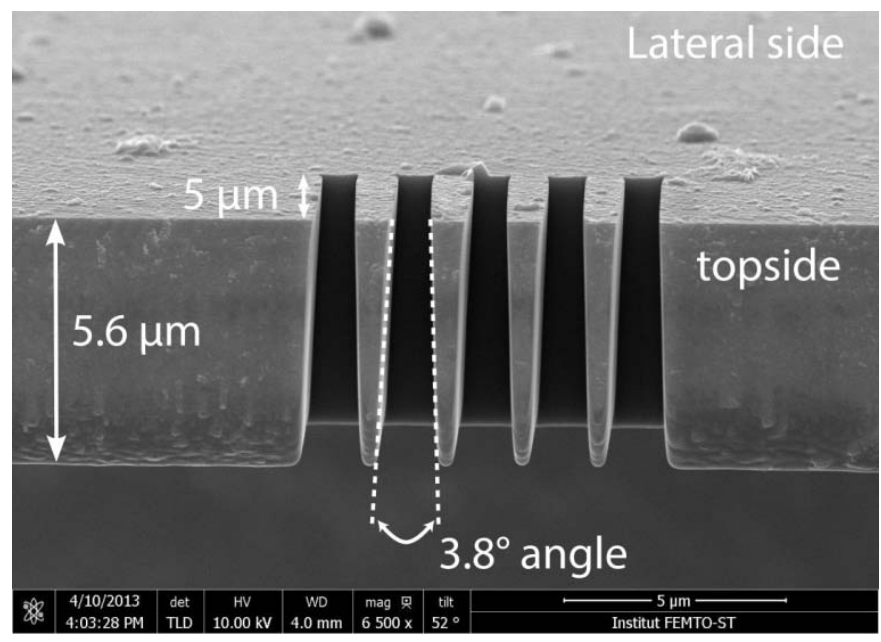

Fig. 4. SEM view of the Bragg grating etched from the lateral side of a ridge by FIB milling. The $14^{\circ}$ tilt from the top part of the ridge allows to see the bottom part of the Bragg grating. The dashed lines show the sidewall angle.

The reflected density spectrum of the ultrashort and laterally etched Bragg grating is measured by means of an optical coherence tomography (OCT) set-up, shown in Fig. 5. A broadband infrared beam is firstly generated with an amplified spontaneous emission (ASE) source in the C-band (HighWave Optical Technologies, Erbium doped fiber broadband source HWT-BS-B1-2). A fiber optic polarizer followed by a polarization controller is connected at the output of the source, in order to inject a broadband polarized light at the input of the structured ridge waveguide. A fiber optic circulator is inserted between the polarization controller and the waveguide entrance with the purpose of measuring the reflected power. Transmitted and reflected optical spectrum densities are finally measured respectively at the output or at the input of the structured waveguides by means of a high resolution optical spectrum analyzer (AP2040A APEX). 


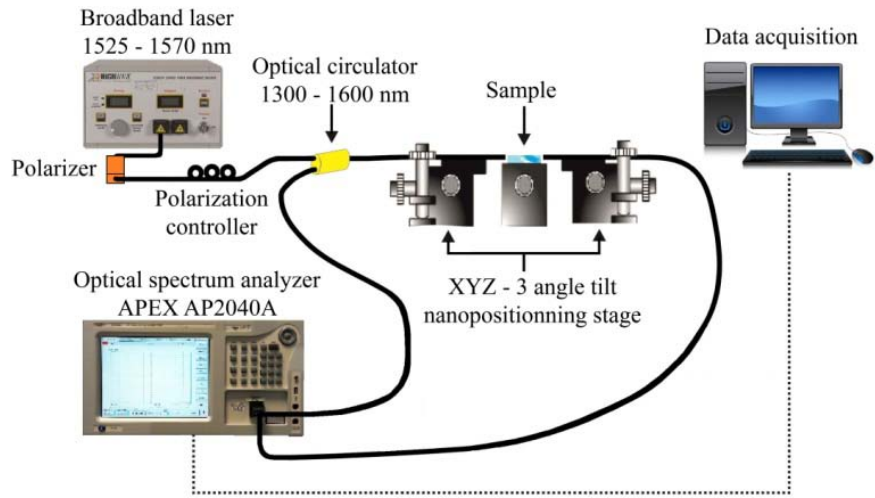

Fig. 5. Experimental set-up for the measurement of the transmission and reflection through the Bragg grating in the $\mathrm{C}^{-}$ band.

Fig. 6.a. shows an example of the reflected optical density spectrum measured at the input of the waveguide. It was measured for a TM-polarized wave propagating in the ridge waveguide with the 4-period Bragg grating shown in Fig. 4. The oscillations seen in the figure correspond to the guided light going back and forth between the input facet and the Bragg grating: the Free Spectral Range of $0.3 \mathrm{~nm}$ is related to the optical cavity length, whilst the finesse enables an evaluation of the reflection coefficient. Further analysis is done by calculating the Fourier transform of the reflected signal, which leads to the impulse response autocorrelation reflected by the device. The result is represented in Fig. 6.b. : the peaks correspond to echoes of the light inside optical cavities. The green and pink lines represent respectively the optical responses measured for the TM and TE polarized guided waves propagating in the ridge waveguide without any grating. The effective group index is deduced from the temporal position of the first reflected peak: it is estimated to be 2.19 for the TE-wave and 2.27 for the TM wave. The relative amplitude of the reflected peaks confirms the estimation of the propagation losses: $1.05 \pm 0.05 \mathrm{~dB} / \mathrm{cm}$ for TE wave and $3.2 \pm 0.1 \mathrm{~dB} / \mathrm{cm}$ for TM wave. The same measurements have been performed once the Bragg grating (Fig. 4) had been inscribed in the ridge. The resulting optical responses are seen in blue (TM wave) and red (TE wave) lines in Fig. 5. Now the reflected peaks are significantly shifted, meaning that the light is no longer reflected by the output facet of the ridge, but rather by the Bragg grating. From the amplitude of the reflected peaks, we are able to evaluate the reflectivity of the grating: $48.1 \%$ for the TE wave and $53.2 \%$ for the TM wave, which is almost three times higher than the Fresnel reflection coefficients. This result is similar to the $50 \%$ reflector proposed by Zhou et al. [8] but with a grating 17 times shorter and which allows the propagation of both polarizations. However, these reflection coefficients are far from the $99.7 \%$ of reflection expected with a perfect Bragg grating, mainly due to imperfections of the grating: the Gaussian shape of the ion beam induces a change of the mark-to-space ratio along the width of ridge, which causes small disorder of the length of the period along the grating.
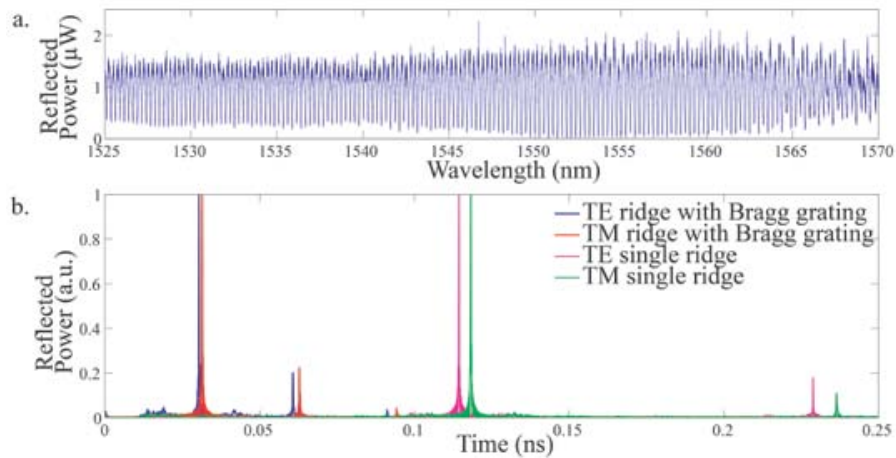

Fig. 6. a. Reflected spectral density measured at the input of the structured ridge waveguide with Bragg gratings (TM wave). b. Fourier transforms of the above spectrum, corresponding to the impulse response correlation of the reflected light in the ridge with and without the Bragg grating for both polarization.

Numerical simulations of the propagation of the electromagnetic field were performed using a 2D-FDTD (Finite Difference Time Domain) code, in order to evaluate the influence of the angle between two consecutive walls in the Bragg grating. The resulting numerical reflection response for TM-polarized mode through the gratings is represented in Fig. 7 as a function of the sidewall angle between two walls of the grating. The scheme of the photonic structure, which served as model for 2D-FDTD simulations is shown in Fig. 8, and the calculated electric field distribution is seen for different sidewall angles. With only 5 grooves of air, a perfect Bragg grating (angle of $0^{\circ}$ ) would reflect $98.4 \%$ of the incident light energy (see the blue line in Fig. 7). With an angle of $2^{\circ}$, the reflectivity stays as high as $97.6 \%$, and still allows sharp bandgap edges, but a red shift of the photonic bandgap can be observed. Higher angles however induce increased radiation losses: it is seen in Fig. 8 that beyond $3^{\circ}$ angles the light is significantly deflected. For $3^{\circ}$ sidewall angles, the maximum of reflection stays higher than $90 \%$ but the slope of the photonic bandgap is not sharp anymore. For sidewall angles over $3.5^{\circ}$, the maximum of reflection decreases down to $80 \%$ at $1660 \mathrm{~nm}$. The theoretical coefficients of reflection at $1.55 \mu \mathrm{m}$ for a $3.8^{\circ}$ angle Bragg grating at $1550 \mathrm{~nm}$ are of $52 \%$ for TE-polarization and $51.85 \%$ for TM-polarization. These simulations are in good agreement with the experimental results. Nevertheless, it is worth noting that a reflectivity as high as $80 \%$ is expected at $1650 \mathrm{~nm}$ with a $3.8^{\circ}$ angle: an enhanced reflectivity at $1550 \mathrm{~nm}$ is thus expected with an optimized grating period. Consequently, there is a considerable interest in developing a fabrication process that yields grooves with sidewall angles very close to $0^{\circ}$, and no larger than $4^{\circ}$. 


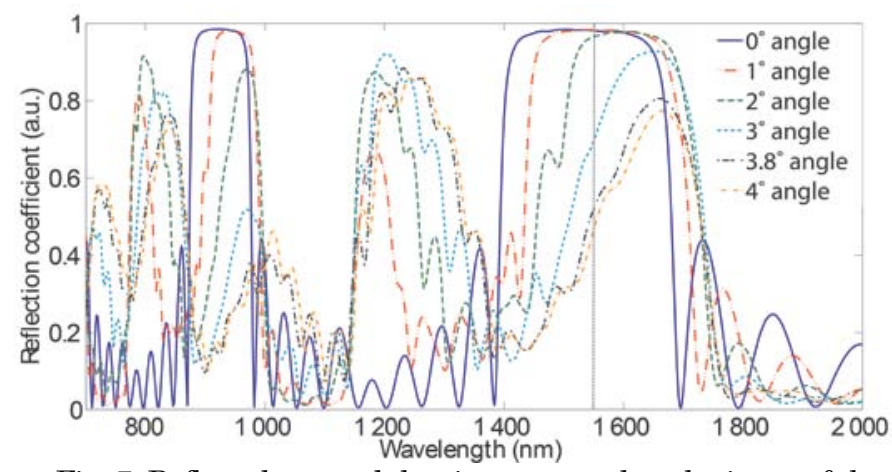

Fig. 7. Reflected spectral density measured at the input of the structured ridge waveguide with Bragg gratings (TM wave). b. Fourier transforms of the above spectrum, corresponding to the impulse response correlation of the reflected light in the ridge with and without the Bragg grating for both polarizations.
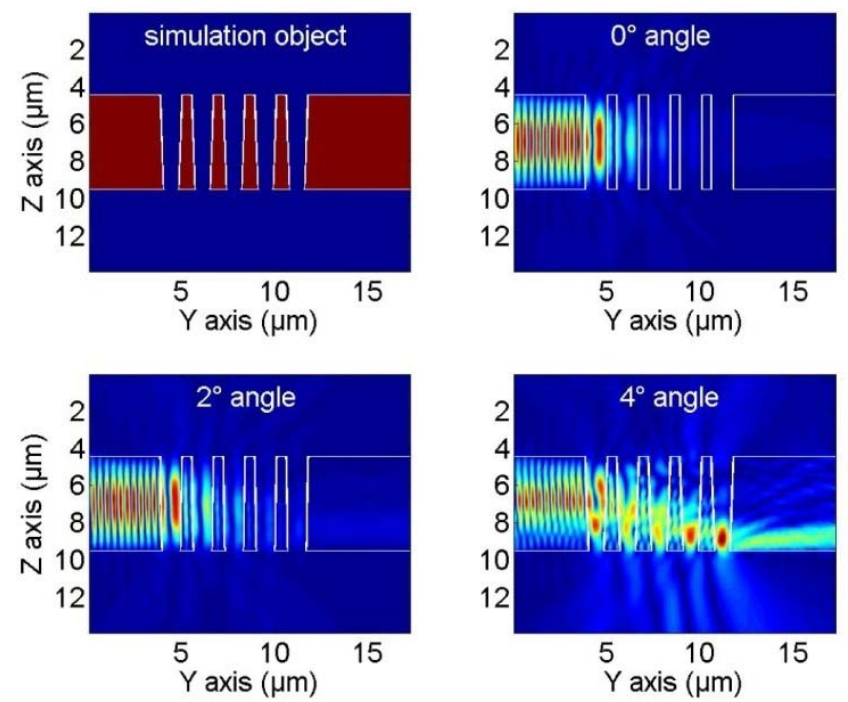

Fig. 8. Theoretical distribution of Ez field in the Bragg grating at $1550 \mathrm{~nm}$ for different sidewall angles.

In summary, we have successfully fabricated and optically characterized extremely short Bragg gratings in Ti-indiffused lithium niobate waveguide, with a total length of $8 \mu \mathrm{m}$. The experimental reflectivity of $48.1 \%$ for the TE wave and $53.2 \%$ for the TM wave are similar to our knowledge to current state of the art of shortest Bragg gratings in lithium niobate, but the active length was severely reduced compared to structures made by photorefraction, enabling to go a step further toward integrated photonic components in lithium niobate.
This work was supported by the ANR Matériaux et Procédés pour les Produits Innovants 2012 under project CHARADES, by the SNIFER DGA RAPID project contract number 112906210, by the Labex ACTION program (contract ANR-11-LABX-01-01) and by the Conseil Régional de Franche-Comté, France. The work was partly supported by the French RENATECH network and its FEMTO-ST technological facility. The authors would like to thank Blandine Guichardaz for helpful support in clean room, and Jean-Charles Beugnot, Thibaut Sylvestre and Rémo Giust for fruitful discussions.

\section{References :}

[1] A. Guarino, G. Poberaj, D. Rezzonico, R. Dell'Innocenti and P. Günter, Nature Photonics, 1, 407-410 (2007).

[2] B.-E. Benkelfat, R. Ferrière, B. Wacogne, P. Mollier, IEEE Photonics and Technology Letters, 14, 1430-1432 (2002).

[3] D. N. Urquidez, S. Stepanov, H. Soto Ortiz, N. Toguzov, I Ilichev, A. Shamray, Applied Physics B 106, 51-56 (2012).

[4] H. Feng, R.F. Tavlykaev and R.V. Ramaswamy, Electronic Letters 35, 1636 (1999).

[5] W. Horn, S. Kroesen, J. Herrmann, J. Imbrock and C. Denz, Optics Express 20, 26922-26928 (2012).

[6] J. Hukriede, D. Kip and E. Kratzig, Journal of Optics A, 2, 481-487 (2000).

[7] G. Ulliac, A. Lecestre, B. Guichardaz, J. Dahdah, F.I. Baida, M.-P. Bernal and N. Courjal, Microelectronic Engineering 97, 185-188 (2012).

[8] Z. Zhou, X. Huang, R. Rao Vanga, Z. Wu, JOSA B 27, 1425-1429 (2010)

[9] N. Courjal, J. Dahdah, G. Ulliac, P. Seviallano, B. Guichardaz and F. Baida, Optics Express 19, 2300823016 (2011).

[10] N. Courjal, B. Guichardaz, G. Ulliac, J.-Y. Rauch, B. Sadani, H.-H. Lu, and M.-P. Bernal, J. Phys. D: Appl. Phys., Vol. 44, 305101 (2011).

[11] I. P. Kaminov, V. Ramaswamy, R. V. Schmidt and E. H. Turner, Applied Physics Letters 24, 622-624 (1974).

[12] H. Hu, R. Ricken and W. Sohler, Optics Express 17, 26, 24261-24268 (2010).

[13] F. Lacour, N. Courjal, M.-P. Bernal, A. Sabac, C. Bainier and M. Spajer, Optical Materials 27,1421-1425 (2005).

[14] G. W. Burr, S. Diziain, and M.-P. Bernal, Optics Express 16, 6302-6316 (2008). 